\title{
From molecules to molecular devices
}

\author{
Wolfgang Linert · Tony Vlcek
}

Received: 7 January 2009/ Accepted: 9 January 2009/Published online: 17 March 2009

(C) Springer-Verlag 2009

Modern chemistry allows us to make new molecules and combine them into materials with unprecedented optical, magnetic or electrical properties. Importantly, we can often trigger abrupt changes in such molecules by external stimuli, making so-called "molecular switches." Sudden changes in the molecular structure are accompanied by changes in the physical properties of the resulting materials. Thus, for example, brief irradiation with a pulse of light switches the spin state of a suitable molecule, triggering profound changes in the magnetism of its crystals, films or nanoparticles. This and other types of "molecular switching" can be utilized, for example, in molecular memory. At its limit, a bit of information could be stored as a spin state of a single magnetochemically active molecule. The duration of such a process is very important: molecular switching should be as fast as possible, preferentially occurring in picoseconds or even faster-comparable with semiconductor electronics. With the advent of nanochemistry, we also need to focus on spatial dimensions. The properties of nanoparticles in the approximate range $10-100 \mathrm{~nm}$ often depend not only on the nature of the constituent atoms or molecules but also on how many there are. Consequently, many important functions of nanomaterials occur only for certain sizes and can be controlled by their environment. Another type of molecular response is chemical; in this case, molecular or nanoparticle properties (for example fluorescence) depend on the chemical composition of the solution. Such species are the basis for sensors that selectively report on a range of species, from metal ions to biomolecules. Clearly, chemistry has a great potential to develop functional chemical systems (so-called molecular devices) that can perform various operations

W. Linert $(\square) \cdot$ T. Vlcek

Vienna University of Technology, Vienna, Austria

e-mail: wlinert@mail.zserv.tuwien.ac.at based on molecular switching. Long ago, nature evolved complex and highly organized molecular systems to perform processes essential for life; the photosynthetic reaction center and the rhodopsin receptors in our eyes are excellent examples of molecular devices for the chemical conversion of optical energy and information, respectively.

At the European level, broad multidisciplinary research in this field is promoted through the programme Cooperation in the Field of Scientific and Technical Research (COST). One of its chemistry actions, D35 (From Molecules to Molecular Devices: Control of Electronic, Photonic, Magnetic and Spintronic Behaviour) focuses on the development of new functional molecules and investigations of their properties and responses to various external perturbations. This COST action runs from 2006 until 2011, supporting collaboration between over seventy European research groups active in diverse fields such as photoelectrochemical and photochemical light-energy conversion, control of electronic excited states of metal complexes and their assemblies, development of new metalbased materials for organic light-emitting diodes, liquid crystal molecular switches, spin crossover phenomena, crystalline and thin-film switchable molecular materials with novel electro-optical properties, and assemblies of photo- or redox active metal complexes and nanoparticles.

This special issue of Chemistry Monthly presents some of the research carried out within the remit of COST action D35 and beyond. The articles focus on metal complexes, polymeric and fullerene-based systems and their photonic, sensing and magnetic functions, as well as the possible use of metal complexes in molecular wires.

Wolfgang Linert

Tony Vlcek

Issue Editors 